\title{
Optimal Bang-Bang Trajectories in Sub-Finsler Problem on the Cartan Group
}

\author{
Yu. Sachkov
}

The Cartan group is the free nilpotent Lie group of step 3, with 2 generators. This paper studies the Cartan group endowed with the left-invariant sub-Finsler $\ell_{\infty}$ norm. We adopt the viewpoint of time-optimal control theory. By Pontryagin maximum principle, all sub-Finsler length minimizers belong to one of the following types: abnormal, bang-bang, singular, and mixed. Bang-bang controls are piecewise controls with values in the vertices of the set of control parameter.

In a previous work, it was shown that bang-bang trajectories have a finite number of patterns determined by values of the Casimir functions on the dual of the Cartan algebra. In this paper we consider, case by case, all patterns of bang-bang trajectories, and obtain detailed upper bounds on the number of switchings of optimal control.

For bang-bang trajectories with low values of the energy integral, we show optimality for arbitrarily large times.

The bang-bang trajectories with high values of the energy integral are studied via a second order necessary optimality condition due to A. Agrachev and R. Gamkrelidze. This optimality condition provides a quadratic form, whose sign-definiteness is related to optimality of bangbang trajectories. For each pattern of these trajectories, we compute the maximum number of switchings of optimal control. We show that optimal bang-bang controls may have not more than 11 switchings. For particular patterns of bang-bang controls, we obtain better bounds. In such a way we improve the bounds obtained in previous works.

On the basis of results of this work we can start to study the cut time along bang-bang trajectories, i.e., the time when these trajectories lose their optimality. This question will be considered in subsequent works.

Keywords: sub-Finsler geometry, optimal control, switchings, bang-bang trajectories

Received October 24, 2018

Accepted December 03, 2018

The research leading to these results has received funding from the Ministry of Education and Science of the Russian Federation in the framework of the project RFMEFI60716X0153.

Yurii Sachkov

yusachkov@gmail.com

A. K. Ailamazyan Program Systems Institute of RAS

ul. Petra I 4a, Veskovo, Pereslavl district, Yaroslavl region, 152021 Russia

RUSSIAN JOURNAL OF NONLINEAR DYNAMICS, 2018, 14(4), 583-593 


\section{Introduction}

Sub-Finsler geometry on Lie groups has received considerable attention during last years due to its applications, especially in geometric group theory and in harmonic analysis, see articles $[4,6,10]$ and introductions of $[16,18]$ for a broad explanation of the reasons and for several references of the state-of-the-art. To our knowledge the term sub-Finsler appears for the first time in paper [11].

In the case of step two nilpotent Lie groups and homogeneous spaces there is a good understanding of sub-Finsler structures (Heisenberg group, flat Martinet case, Grushin plane) after work [16]. On the other hand, a detailed study of the left-invariant sub-Finsler structure on the free nilpotent Lie group of step 3 with 2 generators (called the Cartan group) began in works $[18,19]$. This paper continues those works.

We adopt the viewpoint of time-optimal control theory. Pontryagin maximum principle [13] implies that sub-Finsler length minimizers are of one of the following types: abnormal, bangbang, singular, or mixed (concatenations of finite number of bang and singular arcs). In this work we study optimality of bang-bang trajectories. There is a finite number of patterns of these trajectories described in $[18,19]$, and for each pattern we prove an upper bound on the number of switchings of bang-bang optimal control. The main tool here is a second order necessary optimality condition due to A. Agrachev and R. Gamkrelidze [15].

This work has the following structure. In Section 2 we recall the problem statement and some previously obtained results from $[18,19]$. In Section 3 the second order optimality condition by Agrachev - Gamkrelidze [15] is stated. In Section 4 we prove the main results of this paper: we consider all patterns of bang-bang trajectories, and obtain upper bounds on the number of switchings of the optimal control. Results of Section 4 improve Theorem 6 [18] by giving detailed bounds on the number of switchings for all patterns of bang-bang optimal control. Finally, some concluding remarks are given in Section 5.

\section{Problem statement and previous results}

Consider the 5-dimensional free nilpotent Lie algebra with 2 generators, of step 3 . There exists a basis $L=\operatorname{span}\left(X_{1}, \ldots, X_{5}\right)$ in which the product rule in $L$ takes the form

$$
\left[X_{1}, X_{2}\right]=X_{3}, \quad\left[X_{1}, X_{3}\right]=X_{4}, \quad\left[X_{2}, X_{3}\right]=X_{5}, \quad \operatorname{ad} X_{4}=\operatorname{ad} X_{5}=0 .
$$

The Lie algebra $L$ is called the Cartan algebra, and the corresponding connected simply connected Lie group $M$ is called the Cartan group. We will use the following model:

$$
M=\mathbb{R}_{x, y, z, v, w}^{5},
$$

with the Lie algebra $L$ modeled by left-invariant vector fields on $\mathbb{R}^{5}$

$$
\begin{aligned}
& X_{1}=\frac{\partial}{\partial x}-\frac{y}{2} \frac{\partial}{\partial z}-\frac{x^{2}+y^{2}}{2} \frac{\partial}{\partial w}, \\
& X_{2}=\frac{\partial}{\partial y}+\frac{x}{2} \frac{\partial}{\partial z}+\frac{x^{2}+y^{2}}{2} \frac{\partial}{\partial v}, \\
& X_{3}=\frac{\partial}{\partial z}+x \frac{\partial}{\partial v}+y \frac{\partial}{\partial w},
\end{aligned}
$$




$$
\begin{aligned}
& X_{4}=\frac{\partial}{\partial v}, \\
& X_{5}=\frac{\partial}{\partial w} .
\end{aligned}
$$

The product rule in the Cartan group $M$ in this model is given in [12].

Left-invariant $\ell_{\infty}$ sub-Finsler problem on the Cartan group is stated as the following timeoptimal problem:

$$
\begin{aligned}
& \dot{q}=u_{1} X_{1}+u_{2} X_{2}, \quad q \in M, \quad u \in U=\left\{u \in \mathbb{R}^{2} \mid\|u\|_{\infty} \leqslant 1\right\} \\
& \|u\|_{\infty}=\max \left(\left|u_{1}\right|,\left|u_{2}\right|\right), \\
& q(0)=q_{0}=\operatorname{Id}=(0, \ldots, 0), \quad q(T)=q_{1}, \\
& T \rightarrow \min .
\end{aligned}
$$

Problem (2.1)-(2.3) was considered first in papers [18, 19]. We recall some results of those papers.

Existence of optimal controls follows from Rashevsky - Chow and Filippov theorem [13].

Pontryagin Maximum Principle implies that optimal abnormal controls are constant.

Introduce linear-on-fibers Hamiltonians $h_{i}(\lambda)=\left\langle\lambda, X_{i}\right\rangle, \lambda \in T^{*} M, i=1, \ldots, 5$. A normal extremal $\operatorname{arc} \lambda_{t}, t \in I=(\alpha, \beta) \subset[0, T]$ is called:

- a bang-bang arc if

$$
\operatorname{card}\left\{t \in I \mid h_{1} h_{2}\left(\lambda_{t}\right)=0\right\}<\infty,
$$

- a singular arc if one of the condition holds:

$$
\begin{array}{lll}
h_{1}\left(\lambda_{t}\right) \equiv 0, & t \in I \quad & \left(h_{1} \text {-singular arc }\right), \text { or } \\
h_{2}\left(\lambda_{t}\right) \equiv 0, \quad t \in I & \left(h_{2} \text {-singular arc }\right),
\end{array}
$$

- a mixed arc if it consists of a finite number of bang-bang and singular arcs.

Singular controls have one of components constantly equal to 1 or -1 , thus they are optimal. The fix-time attainable set along singular trajectories was explicitly described and was shown to be semi-algebraic.

Bang-bang extremal trajectories satisfy the Hamiltonian system with the Hamiltonian function $H=\left|h_{1}\right|+\left|h_{2}\right|$ :

$$
\left\{\begin{array}{l}
\dot{h}_{1}=-s_{2} h_{3}, \\
\dot{h}_{2}=s_{1} h_{3}, \\
\dot{h}_{3}=s_{1} h_{4}+s_{2} h_{5}, \\
\dot{h}_{4}=\dot{h}_{5}=0, \\
\dot{q}=s_{1} X_{1}+s_{2} X_{2} .
\end{array}\right.
$$

The dual of the Lie algebra $L^{*}=T_{\mathrm{Id}}^{*} M$ has Casimir functions $h_{4}, h_{5}, E=\frac{h_{3}^{2}}{2}+h_{1} h_{5}-h_{2} h_{4}$, thus Hamiltonian system (2.4) has integrals $h_{4}, h_{5}, E$, and $H$.

The mapping $(\lambda, q) \mapsto(k \lambda, q), k>0$, preserves extremal trajectories, thus we can consider only the reduced case

$$
H(\lambda) \equiv 1
$$


With the use of the coordinate $\theta \in S^{1}=\mathbb{R} / 2 \pi \mathbb{Z}$ :

$$
h_{1}=\operatorname{sgn}(\cos \theta) \cos ^{2} \theta, \quad h_{2}=\operatorname{sgn}(\sin \theta) \sin ^{2} \theta,
$$

the vertical part of Hamiltonian system (2.4) reduces to the following system:

$$
\left\{\begin{array}{l}
\dot{\theta}=\frac{h_{3}}{|\sin 2 \theta|}, \quad \theta \neq \frac{\pi n}{2}, \\
\dot{h}_{3}=s_{1} h_{4}+s_{2} h_{5}, \quad s_{1}=\operatorname{sgn} \cos \theta, \quad s_{2}=\operatorname{sgn} \sin \theta .
\end{array}\right.
$$

Consider the cylinder

$$
C=T_{q_{0}}^{*} M \cap\{H=1\} .
$$

In work [18] it was shown that bang-bang trajectories can be represented as images of an exponential mapping: $\{q(t)\}=\operatorname{Exp}(\lambda, t), \lambda \in C, t>0$. The exponential mapping is singlevalued for generic $\lambda \in C$, and is multi-valued for certain special subsets of $C$, see [18].

System (2.5) is preserved by the group of symmetries of the square $\left\{\left(h_{1}, h_{2}\right) \in \mathbb{R}^{2}|| h_{1} \mid+\right.$ $\left.+\left|h_{2}\right|=1\right\}$. Thus in the study of system (2.5) we can restrict ourselves by the case $h_{4} \geqslant h_{5} \geqslant 0$. This group of symmetries reduces the cylinder $C$ to the fundamental domain of the group $\left\{\lambda \in C \mid h_{4} \geqslant h_{5} \geqslant 0\right\}$. Further, this fundamental domain admits a stratification by invariant subsets of the Hamiltonian system (2.5):

$$
\begin{aligned}
& \left\{\lambda \in C \mid h_{4} \geqslant h_{5} \geqslant 0\right\}=\cup_{i=1}^{4} C^{i}, \\
& C^{1}=\left\{\lambda \in C \mid h_{4}>h_{5}>0\right\}, \\
& C^{2}=\left\{\lambda \in C \mid h_{4}>h_{5}=0\right\}, \\
& C^{3}=\left\{\lambda \in C \mid h_{4}=h_{5}>0\right\}, \\
& C^{4}=\left\{\lambda \in C \mid h_{4}=h_{5}=0\right\} .
\end{aligned}
$$

Further, we have the following stratifications:

$$
\begin{aligned}
& C^{1}=\cup_{i=1}^{8} C_{i}^{1}, \\
& C_{1}^{1}=E^{-1}\left(-h_{4}\right), \quad C_{2}^{1}=E^{-1}\left(-h_{4},-h_{5}\right), \quad C_{3}^{1}=E^{-1}\left(-h_{5}\right), \quad C_{4}^{1}=E^{-1}\left(-h_{5}, h_{5}\right), \\
& C_{5}^{1}=E^{-1}\left(h_{5}\right), \quad C_{6}^{1}=E^{-1}\left(h_{5}, h_{4}\right), \quad C_{7}^{1}=E^{-1}\left(h_{4}\right), \quad C_{8}^{1}=E^{-1}\left(h_{4},+\infty\right), \\
& C^{2}=\cup_{i=1}^{6} C_{i}^{2}, \\
& C_{1}^{2}=E^{-1}\left(-h_{4}\right), \quad C_{2}^{2}=E^{-1}\left(-h_{4}, 0\right), \quad C_{3}^{2}=E^{-1}(0), \\
& C_{4}^{2}=E^{-1}\left(0, h_{4}\right), \quad C_{5}^{2}=E^{-1}\left(h_{4}\right), \quad C_{6}^{2}=E^{-1}\left(h_{4},+\infty\right), \\
& C^{3}=\cup_{i=1}^{4} C_{i}^{3}, \\
& C_{1}^{3}=E^{-1}\left(-h_{4}\right), \quad C_{2}^{3}=E^{-1}\left(-h_{4}, 0\right), \quad C_{3}^{3}=E^{-1}\left(h_{4}\right), \quad C_{4}^{3}=E^{-1}\left(h_{4},+\infty\right), \\
& C^{4}=C_{1}^{4} \cup C_{2}^{4}, \\
& C_{1}^{4}=E^{-1}(0), \quad C_{2}^{4}=E^{-1}(0,+\infty) .
\end{aligned}
$$

In paper [19] was obtained the following optimality result for bang-bang trajectories with low energy $E$.

Theorem 1 ([19, Theorem 2]). If a bang-bang extremal $\lambda_{t}, t \in[0,+\infty)$, satisfies the inequality

$$
\min \left(-\left|h_{4}\right|,-\left|h_{5}\right|\right)<E \leqslant \max \left(-\left|h_{4}\right|,-\left|h_{5}\right|\right)
$$

then it is optimal. 


\section{Theorem by Agrachev-Gamkrelidze}

We obtain an upper bound on the number of switchings on optimal bang-bang trajectories via the following theorem due to A. Agrachev and R. Gamkrelidze.

Theorem 2 ([15, 16]). Let $(q(\cdot), u(\cdot))$ be an extremal pair for problem $(2.1)-(2.3)$ and let $\lambda$. be an extremal lift of $q(\cdot)$. Assume that $\lambda$. is the unique extremal lift of $q(\cdot)$, up to multiplication by a positive scalar. Assume that there exist $0=t_{0}<t_{1}<t_{2}<\ldots<t_{k}<\tau_{k+1}=T$ and $u^{0}, \ldots, u^{k} \in U$ such that $u(\cdot)$ is constantly equal to $u^{j}$ on $\left(\tau_{j}, \tau_{j+1}\right)$ for $j=0, \ldots, k$.

Fix $j=1, \ldots, k$. For $i=0, \ldots, k$ let $Y_{i}=u_{1}^{i} X_{1}+u_{2}^{i} X_{2}$ and define recursively the operators

$$
\begin{aligned}
& P_{j}=P_{j-1}=\operatorname{Id}_{\operatorname{Vec}(M)}, \\
& P_{i}=P_{i-1} \circ e^{\left(t_{i}-t_{i-1}\right) \operatorname{ad} Y_{i-1}}, \quad i=j+1, \ldots, k, \\
& P_{i}=P_{i+1} \circ e^{-\left(t_{i+2}-t_{i+1}\right) \text { ad } Y_{i+1}}, \quad i=0, \ldots, j-2 .
\end{aligned}
$$

Define the vector fields

$$
Z_{i}=P_{i}\left(Y_{i}\right), \quad i=0, \ldots, k
$$

Let $Q$ be the quadratic form

$$
Q(\alpha)=\sum_{0 \leqslant i<l \leqslant k} \alpha_{i} \alpha_{l}\left\langle\lambda_{t_{j}},\left[Z_{i}, Z_{l}\right]\left(q\left(t_{j}\right)\right)\right\rangle
$$

defined on the space

$$
W=\left\{\alpha=\left(\alpha_{0}, \ldots, \alpha_{k}\right) \in \mathbb{R}^{k+1} \mid \sum_{i=0}^{k} \alpha_{i}=0, \quad \sum_{i=0}^{k} \alpha_{i} Z_{i}\left(q\left(t_{j}\right)\right)=0\right\} .
$$

If $Q$ is not negative-semidefinite, then $q(\cdot)$ is not optimal.

\section{Bounds on the number of switchings}

Now we obtain bounds on the number of switchings for bang-bang optimal trajectories $\operatorname{Exp}(\lambda, t)$ with $\lambda \in \cup_{i=1}^{4} C^{i}$, case by case.

\subsection{Case $\lambda \in C^{1}$}

In the case $\lambda \in C^{1}$ system (2.5) has phase portrait given in Fig. 1.

\subsubsection{Low values of integral $E$}

Theorem 1 implies the following statement.

Corollary 1. If $\lambda \in C_{1}^{1} \cup C_{2}^{1} \cup C_{3}^{1}$, then the trajectory $\operatorname{Exp}(\lambda, t), t \in[0,+\infty)$, is optimal.

\subsubsection{High values of integral $E$}

We apply Theorem 2 and obtain the following upper bounds on the number of switchings on optimal bang-bang trajectories. An example of detailed computation on the basis of Theorem 2 is given in the proof of Theorem 5 [19].

RUSSIAN JOURNAL OF NONLINEAR DYNAMICS, 2018, 14(4), 583-593 


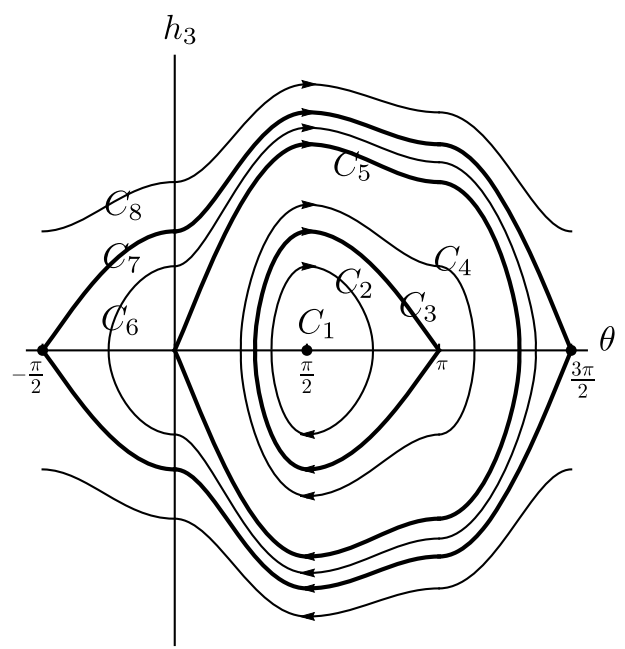

Fig. 1. Phase portrait of system (2.5) in case $\lambda \in C^{1}$.

Theorem 3. Let $\lambda \in \cup_{i=4}^{8} C_{i}^{1}$. Then the bang-bang trajectory $\operatorname{Exp}(\lambda, t)$ with $k$ switchings is not optimal, where $k$ is given by the following tables:

- $\lambda \in C_{4}^{1} \cup C_{5}^{1} \Rightarrow$ Table 1 ,

- $\lambda \in C_{6}^{1} \Rightarrow$ Table 2 ,

- $\lambda \in C_{7}^{1} \Rightarrow$ Table 3 ,

- $\lambda \in C_{8}^{1} \Rightarrow$ Table 4 .

REMARK 1. We explain now how Tables 1-4 should be read.

Consider Table 1. The first line - Start — gives the values of $\left(u_{1}(0), u_{2}(0)\right)=\left(\operatorname{sgn} h_{1}(0), \operatorname{sgn} h_{2}(0)\right)$ and, if necessary, the signs of $h_{3}(0)$ as a lower index. For example, the first column of Table 1 corresponds to $\left(u_{1}(0), u_{2}(0)\right)=\left(\operatorname{sgn} h_{1}(0), \operatorname{sgn} h_{2}(0)\right)=(+1,+1)$. The second column of Table 1 corresponds to the initial values $\left(u_{1}(0), u_{2}(0)\right)=\left(\operatorname{sgn} h_{1}(0), \operatorname{sgn} h_{2}(0)\right)=(-1,+1)$ and $\operatorname{sgn} h_{3}(0)=+1$. The second line of Table 1 gives the number of switchings $k$ for the corresponding $\lambda \in C_{4}^{1} \cup C_{5}^{1}$ such that the bang-bang trajectory $\operatorname{Exp}(\lambda, t)$ is not optimal. Similar agreement is applied for Tables 2, 4 .

Table 3 should be read as follows. Consider, e.g., entry $N=10$ of Table 3 . The sequence of signs $(+,-)++-$ has the following meaning:

- the signs $(+,-)$ determine the initial control $\left(u_{1}(0), u_{2}(0)\right)=\left(\operatorname{sgn} h_{1}(0), \operatorname{sgn} h_{2}(0)\right)=(+1,-1)$,

- the subsequent signs ++- determine the signs of $h_{3}(t)$ between switchings of control, i.e.,

$$
\begin{aligned}
& -\operatorname{sgn} h_{3}(t)=+1, t \in\left[0, t_{1}\right] ; \\
& -\operatorname{sgn} h_{3}(t)=+1, t \in\left[t_{1}, t_{2}\right] ; \\
& -\operatorname{sgn} h_{3}(t)=-1, t \in\left[t_{2}, T\right],
\end{aligned}
$$

where $t_{1}, t_{2}$ are switching times at which $h_{3}(t)$ vanishes.

The number 9 for entry $N=10$ of Table 3 gives the number of switchings of a non-optimal bang-bang control.

The same agreement on reading similar tables is used in subsequent subsections.

The below cases $\lambda \in_{i=2}^{4} C^{i}$ are considered similarly to the above case $\lambda \in C^{1}$. 
Table 1. $\lambda \in C_{4}^{1} \cup C_{5}^{1}$

\begin{tabular}{|c||c|c|c|c|}
\hline Start & $(+,+)$ & $(-,+)_{+}$ & $(-,-)$ & $(-,+)_{-}$ \\
\hline$k$ & 8 & 9 & 9 & 8 \\
\hline
\end{tabular}

Table 2. $\lambda \in C_{6}^{1}$

\begin{tabular}{|c||c|c|c|c|c|c|}
\hline Start & $(+,+)_{+}$ & $(-,+)_{+}$ & $(-,-)$ & $(-,+)_{-}$ & $(+,+)_{-}$ & $(+,-)$ \\
\hline$k$ & 9 & 10 & 10 & 10 & 9 & 11 \\
\hline
\end{tabular}

Table 3. $\lambda \in C_{7}^{1}$

\begin{tabular}{||r|l|r||r|l|r||r|l|r||r|l|r||}
\hline$N$ & Start & $k$ & $N$ & Start & $k$ & $N$ & Start & $k$ & $N$ & Start & $k$ \\
\hline \hline 1 & $(+,+)+++$ & 9 & 9 & $(+,-)+++$ & 8 & 17 & $(-,+)+++$ & 8 & 25 & $(-,-)+++$ & 8 \\
\hline 2 & $(+,+)++-$ & 9 & 10 & $(+,-)++-$ & 9 & 18 & $(-,+)++-$ & 9 & 26 & $(-,-)++-$ & 8 \\
\hline 3 & $(+,+)+-+$ & 8 & 11 & $(+,-)+-+$ & 11 & 19 & $(-,+)+-+$ & 9 & $27 \mathrm{a}$ & $(-,-)+-++$ & 10 \\
\hline 4 & $(+,+)+--$ & 8 & 12 & $(+,-)+--$ & 9 & 20 & $(-,+)+--$ & 7 & $27 \mathrm{~b}$ & $(-,-)+-+-$ & 11 \\
\hline 5 & $(+,+)-++$ & 9 & 13 & $(+,-)-++$ & 8 & 21 & $(-,+)-++$ & 10 & 28 & $(-,-)+--$ & 8 \\
\hline 6 & $(+,+)-+-$ & 9 & 14 & $(+,-)-+-$ & 12 & $22 \mathrm{a}$ & $(-,+)-+-+$ & 12 & 29 & $(-,-)-++$ & 7 \\
\hline 7 & $(+,+)--+$ & 10 & $15 \mathrm{a}$ & $(+,-)--++$ & 9 & $22 \mathrm{~b}$ & $(-,+)-+--$ & 11 & 30 & $(-,-)-+-$ & 7 \\
\hline 8 & $(+,+)---$ & 9 & $15 \mathrm{~b}$ & $(+,-)--+-$ & 10 & 23 & $(-,+)--+$ & 10 & 31 & $(-,-)--+$ & 7 \\
\hline & & & 16 & $(+,-)---$ & 8 & 24 & $(-,+)---$ & 8 & 32 & $(-,-)---$ & 7 \\
\hline
\end{tabular}

Table 4. $\lambda \in C_{8}^{1}$

\begin{tabular}{|c||c|c|c|c|}
\hline Start & $(+,-)$ & $(+,+)$ & $(-,+)$ & $(-,-)$ \\
\hline$k$ & 8 & 8 & 6 & 6 \\
\hline
\end{tabular}

\subsection{Case $\lambda \in C^{2}$}

In the case $\lambda \in C^{2}$ system (2.5) has phase portrait given in Fig. 2.

\subsubsection{Low values of integral $E$}

Theorem 1 implies the following statement.

Corollary 2. If $\lambda \in C_{1}^{2} \cup C_{2}^{2} \cup C_{3}^{2}$, then the trajectory $\operatorname{Exp}(\lambda, t), t \in[0,+\infty)$, is optimal.

\subsubsection{High values of integral $E$}

Theorem 4. Let $\lambda \in \cup_{i=4}^{2} C_{i}^{2}$. Then the bang-bang trajectory $\operatorname{Exp}(\lambda, t)$ with $k$ switchings is not optimal, where $k$ is given by the following tables:

- $\lambda \in C_{4}^{2} \Rightarrow$ Table 5 ,

- $\lambda \in C_{6}^{2} \Rightarrow$ Table 6 . 


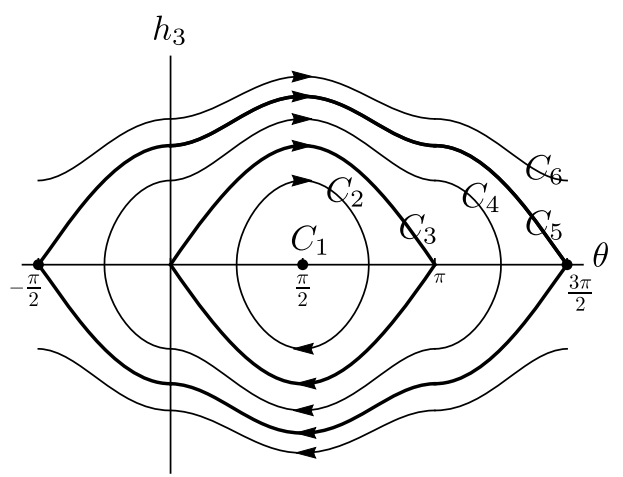

Fig. 2. Phase portrait of system (2.5) in case $\lambda \in C^{2}$.

Table 5. $\lambda \in C_{4}^{2}$

\begin{tabular}{|c||c|c|c|c|c|c|}
\hline Start & $(+,+)_{+}$ & $(-,+)_{+}$ & $(-,-)$ & $(-,+)_{-}$ & $(+,+)_{-}$ & $(+,-)$ \\
\hline$k$ & 8 & 9 & 8 & 8 & 9 & 8 \\
\hline
\end{tabular}

Table 6. $\lambda \in C_{6}^{2}$

\begin{tabular}{|c||c|c|c|c|}
\hline Start & $(+,+)$ & $(-,+)$ & $(-,-)$ & $(+,-)$ \\
\hline$k$ & 7 & 6 & 6 & 7 \\
\hline
\end{tabular}

4.3. Case $\lambda \in C^{3}$

In the case $\lambda \in C^{3}$ system (2.5) has phase portrait given in Fig. 3.

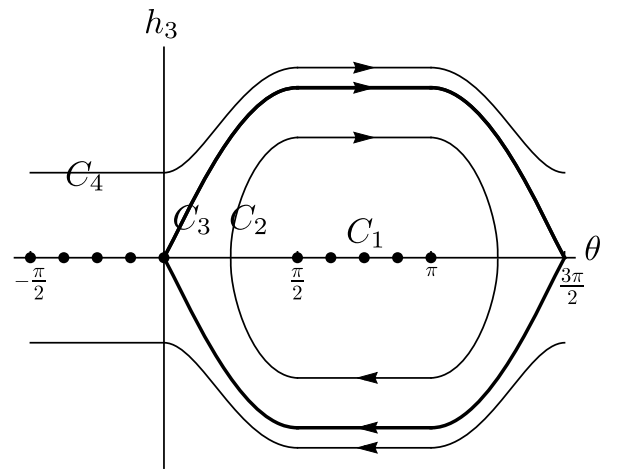

Fig. 3. Phase portrait of system (2.5) in case $\lambda \in C^{3}$.

\subsubsection{Low values of integral $E$}

Theorem 1 implies the following statement.

Corollary 3. If $\lambda \in C_{1}^{3}$, then the trajectory $\operatorname{Exp}(\lambda, t), t \in[0,+\infty)$, is optimal. 


\subsubsection{High values of integral $E$}

Theorem 5. Let $\lambda \in \cup_{i=2}^{4} C_{i}^{3}$. Then the bang-bang trajectory $\operatorname{Exp}(\lambda, t)$ with $k$ switchings is not optimal, where $k$ is given by the following tables:

- $\lambda \in C_{2}^{3} \cup C_{3}^{3} \Rightarrow$ Table 7 ,

- $\lambda \in C_{4}^{3} \Rightarrow$ Table 8 .

Table 7. $\lambda \in C_{2}^{3} \cup C_{3}^{3}$
\begin{tabular}{|c||c|c|c|c|}
\hline Start & $(+,+)$ & $(-,+)_{+}$ & $(-,-)$ & $(-,+)_{-}$ \\
\hline$k$ & 7 & 6 & 7 & 6 \\
\hline
\end{tabular}

Table 8. $\lambda \in C_{3}^{4}$
\begin{tabular}{|c||c|c|c|c|}
\hline Start & $(+,+)$ & $(-,+)$ & $(-,-)$ & $(+,-)$ \\
\hline$k$ & 7 & 6 & 7 & 7 \\
\hline
\end{tabular}

\subsection{Case $\lambda \in C^{4}$}

In the case $\lambda \in C^{4}$ system (2.5) has phase portrait given in Fig. 4.

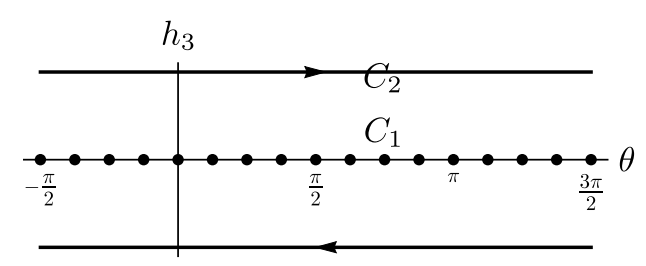

Fig. 4. Phase portrait of system (2.5) in case $\lambda \in C^{4}$.

\subsubsection{Low values of integral $E$}

Theorem 1 implies the following statement.

Corollary 4. If $\lambda \in C_{1}^{4}$, then the trajectory $\operatorname{Exp}(\lambda, t), t \in[0,+\infty)$, is optimal.

\subsubsection{High values of integral $E$}

Theorem 6. Let $\lambda \in C_{2}^{4}$. Then the bang-bang trajectory $\operatorname{Exp}(\lambda, t)$ with $k=7$ switchings is not optimal.

\section{Conclusion}

An obvious next question that arises after the upper bounds on the number of switchings of optimal bang-bang control is the following one: when exactly do the bang-bang trajectories lose their optimality? That is, we would like to describe the cut time along bang-bang trajectories. 
We hope that this is possible by (extension of) the symmetry method applied successfully for description of cut time in several sub-Riemannian and Riemannian problems [20-24]. This question will be studied in forthcoming papers.

\section{References}

[1] Boscain, U., Chambrion, Th., and Charlot, G., Nonisotropic 3-Level Quantum Systems: Complete Solutions for Minimum Time and Minimum Energy, Discrete Contin. Dyn. Syst. Ser. B, 2005, vol.5, no. 4, pp. 957-990.

[2] Berestovskii, V. N., Homogeneous Manifolds with an Intrinsic Metric: 2, Siberian Math. J., 1989, vol. 30, no. 2, pp. 180-191; see also: Sibirsk. Mat. Zh., 1989, vol. 30, no. 2, pp. 14-28, 225.

[3] Berestovskii, V. N., The Structure of Locally Compact Homogeneous Spaces with an Intrinsic Metric, Siberian Math. J., 1989, vol.30, no.1, pp.16-25; see also: Sibirsk. Mat. Zh., 1989, vol.30, no.1, pp. 23-34.

[4] Breuillard, E. and Le Donne, E., On the Rate of Convergence to the Asymptotic Cone for Nilpotent Groups and Sub-Finsler Geometry, Proc. Natl. Acad. Sci. USA, 2013, vol.110, no. 48, pp. 1922019226 .

[5] Clelland, J. N. and Moseley, Ch. G., Sub-Finsler Geometry in Dimension Three, Differential Geom. Appl., 2006, vol. 24, no. 6, pp. 628-651.

[6] Cowling, M. G. and Martini, A., Sub-Finsler Geometry and Finite Propagation Speed, in Trends in Harmonic Analysis, M. A. Picardello (Ed.), Springer INdAM Ser., vol. 3, Milan: Springer, 2013 , pp. $147-205$.

[7] Clelland, J.N., Moseley, Ch.G., and Wilkens, G.R., Geometry of Sub-Finsler Engel Manifolds, Asian J. Math., 2007, vol11, no.4, pp.699-726.

[8] Hakavuori, E. and Le Donne, E., Blowups and Blowdowns of Geodesics in Carnot Groups, arXiv:1806.09375 (2018).

[9] Le Donne, E., A Metric Characterization of Carnot Groups, Proc. Amer. Math. Soc., 2015, vol. 143, no. 2, pp. 845-849.

[10] Pansu, P., Métriques de Carnot-Carathéodory et quasiisométries des espaces symétriques de rang un, Ann. of Math. (2), 1989, vol.129, no.1, pp.1-60.

[11] López, C. and Martínez, E., Sub-Finslerian Metric Associated to an Optimal Control System, SIAM J. Control Optim., 2000, vol. 39, pp. 798-811.

[12] Sachkov, Yu. L., Exponential Mapping in Generalized Dido's Problem, Sb. Math., 2003, vol. 194, no. 9, pp. 1331-1359; see also: Mat. Sb., 2003, vol.194, no. 9, pp. 63-90.

[13] Agrachev, A. A. and Sachkov, Yu. L., Control Theory from the Geometric Viewpoint, Encyclopaedia Math. Sci., vol. 87, Berlin: Springer, 2004.

[14] Pontryagin, L.S., Boltyanskii, V. G., Gamkrelidze, R. V., and Mishchenko, E. F., The Mathematical Theory of Optimal Processes, New York: Wiley, 1962.

[15] Agrachev, A.A. and Gamkrelidze, R. V., Symplectic Geometry for Optimal Control, in Nonlinear Controllability and Optimal Control, H. J.Sussmann (Ed.), Monogr. Textbooks Pure Appl. Math., vol. 133, New York: Dekker, 1990, pp. 263-277.

[16] Barilari, D., Boscain, U., Le Donne, E., and Sigalotti, M., Sub-Finsler Structures from the TimeOptimal Control Viewpoint for Some Nilpotent Distributions, J. Dyn. Control Syst., 2017, vol. 23, no. 3, pp. 547-575.

[17] Gantmacher, F.R., The Theory of Matrices: In 2 Vols., New York: Chelsea, 1959.

[18] Ardentov, A., Le Donne, E., and Sachkov, Yu., A Sub-Finsler Problem on the Cartan Group, Tr. Mat. Inst. Steklova, 2019, accepted.

[19] Ardentov, A., Le Donne, E., and Sachkov, Yu., Sub-Finsler Geodesics on the Cartan Group, Regul. Chaotic Dyn., 2019, accepted. 
[20] Sachkov, Yu., Conjugate and Cut Time in the Sub-Riemannian Problem on the Group of Motions of a Plane, ESAIM Control Optim. Calc. Var., 2010, vol.16, no. 4, pp. 1018-1039.

[21] Ardentov, A. A. and Sachkov, Yu. L., Cut Time in Sub-Riemannian Problem on Engel Group, ESAIM Control Optim. Calc. Var., 2015, vol. 21, no.4, pp. 958-988.

[22] Podobryaev, A. V. and Sachkov, Yu. L., Cut Locus of a Left Invariant Riemannian Metric on $S O(3)$ in the Axisymmetric Case, J. Geom. Phys., 2016, vol. 110, pp. 436-453.

[23] Butt, Y. A., Sachkov, Yu. L., and Bhatti, A. I., Cut Locus and Optimal Synthesis in Sub-Riemannian Problem on the Lie Group SH(2), J. Dyn. Control Syst., 2017, vol. 23, no. 1, pp. 155-195.

[24] Podobryaev, A. V. and Sachkov, Yu. L., Symmetric Riemannian Problem on the Group of Proper Isometries of Hyperbolic Plane, J. Dyn. Control Syst., 2018, vol. 24, no. 3, pp. 391-423. 